\title{
RESEARCH OF NONLINEAR VIBRATIONS OF ORTHOTROPIC PLATES WITH A COMPLEX FORM
}

\author{
L. V. KURPA, T. V. SHMATKO, AND O. G. ONUFRIENKO \\ Received 30 November 2004; Revised 27 May 2005; Accepted 7 July 2005
}

This paper deals with effects of large amplitude on the free and forced flexural vibrations of elastic orthotropic plates of arbitrary shape. $R$-function method (RFM) is applied to obtain the basis functions need for expansion of sought solution into Fourier series. The initial nonlinear system of differential equations with partial derivatives is reduced to system of ordinary nonlinear differential equations by Galerkin procedure. The solvingobtained system is carried out by Runge-Kutta or Galerkin methods. The numerical results for the plate of complex form and also rectangular form and different boundary conditions have been presented and compared with other known results.

Copyright (c) 2006 L. V. Kurpa et al. This is an open access article distributed under the Creative Commons Attribution License, which permits unrestricted use, distribution, and reproduction in any medium, provided the original work is properly cited.

\section{Introduction}

The large amplitude vibration of orthotropic plates was investigated by many scientists $[3,4,8,9,11]$. However most papers are devoted to research of nonlinear vibrations of plates and shallow shells with simple enough form. There are only few works in which the plates and shells, with a shape different from rectangle, circle, ring, or ellipse, are considered. From the authors' point of view, deficiency of such works is connected with difficulties of construction of analytical expressions for basic functions. These functions are needed to reduce a nonlinear system of differential equations with partial derivatives to a system of the ordinary differential equations for time. One of the universal approaches, which can be used for solving this problem, is founded on the usage of the $R$-functions theory $[12,15]$. This theory allows constructing a complete set of the coordinate functions for different types of boundary conditions and practically of an arbitrary domain. Many papers $[7,12-14,16]$ showed the use of the $R$-functions theory for investigation of linear vibrations of plates and shallow shells with different plan forms, a curvature and different types of boundary conditions. In this paper the $R$-functions theory together with variational methods is applied to research of nonlinear vibrations of orthotropic plates of an arbitrary plan form and different types of boundary conditions. 
2 Research of nonlinear vibrations of orthotropic plates

\section{Mathematical statement of the problem}

Thin orthotropic plates with geometric-type nonlinearity subjected to periodic lateral loading are considered. It is assumed that the material of the plate is of uniform thickness and specially orthotropic, with the principal axes of orthotropy being parallel to the $x$ and $y$ directions. Under the assumption that the effect of both the longitudinal and rotatory inertia forces can be neglected, the basic equations governing the nonlinear vibrations of plates can be reduced to the following set of equations $[19,20]$ :

$$
\begin{gathered}
L_{11} u+L_{12} v=N l_{1}(w), \\
L_{21} u+L_{22} v=N l_{2}(w), \\
L_{31} w=N l_{3}(u, v, w)+12\left(1-\mu_{1} \mu_{2}\right) \bar{q}-\lambda^{2} \frac{\partial^{2} w}{\partial t^{2}},
\end{gathered}
$$

where $\xi=x / a, \eta=y / a, \bar{w}=w / h, \bar{u}=u a / h^{2}, \bar{v}=v a / h^{2}$,

$$
\begin{gathered}
L_{11}=C_{1} \frac{\partial^{2}}{\partial \xi^{2}}+C_{2} \frac{\partial^{2}}{\partial \eta^{2}}, \quad L_{12}=L_{21}=C_{3} \frac{\partial^{2}}{\partial \xi \partial \eta}, \quad L_{22}=C_{2} \frac{\partial^{2}}{\partial \xi^{2}}+\frac{\partial^{2}}{\partial \eta^{2}}, \\
L_{31}=C_{1} \frac{\partial^{4}}{\partial \xi^{4}}+2 C_{3} \frac{\partial^{4}}{\partial \xi^{2} \partial \eta^{2}}+\frac{\partial^{4}}{\partial \eta^{4}} \\
N l_{1}(w)=-\left(\frac{1}{2} \frac{\partial}{\partial \xi}\left(C_{1}\left(\frac{\partial w}{\partial \xi}\right)^{2}+\mu_{1}\left(\frac{\partial w}{\partial \eta}\right)^{2}\right)+C_{2} \frac{\partial}{\partial \eta}\left(\frac{\partial w}{\partial \xi} \frac{\partial w}{\partial \eta}\right)\right), \\
N l_{2}(w)=-\left(\frac{1}{2} \frac{\partial}{\partial \eta}\left(\left(\frac{\partial w}{\partial \eta}\right)^{2}+\mu_{1}\left(\frac{\partial w}{\partial \xi}\right)^{2}\right)+C_{2} \frac{\partial}{\partial \xi}\left(\frac{\partial w}{\partial \xi} \frac{\partial w}{\partial \eta}\right)\right), \\
N l_{3}(u, v, w)=12\left(1-\mu_{1} \mu_{2}\right)\left(N_{\xi} \frac{\partial^{2} w}{\partial \xi^{2}}+2 T \frac{\partial^{2} w}{\partial \xi \partial \eta}+N_{\eta} \frac{\partial^{2} w}{\partial \eta^{2}}\right), \\
C_{1}=\frac{E_{1}}{E_{2}}, \quad C_{2}=\frac{G\left(1-\mu_{1} \mu_{2}\right)}{E_{2}}, \quad C_{3}=\frac{G\left(1-\mu_{1} \mu_{2}\right)}{E_{2}}+\mu_{1}=C_{2}+\mu_{1} .
\end{gathered}
$$

In (2.3) value $\lambda^{2}$ is defined as

$$
\lambda^{2}=\frac{\gamma}{g} \frac{a^{4} \cdot 12\left(1-\mu_{1} \mu_{2}\right)}{E_{2} h^{2}} .
$$

And the nondimensional expressions for $N_{\xi}, N_{\eta}$, T have the following form:

$$
\begin{gathered}
N_{\xi}=\frac{C_{1}}{1-\mu_{1} \mu_{2}} \cdot\left(\frac{\partial u}{\partial \xi}+\mu_{2} \frac{\partial v}{\partial \eta}\right)+\frac{C_{1}}{2\left(1-\mu_{1} \mu_{2}\right)} \cdot\left(\left(\frac{\partial \bar{w}}{\partial \xi}\right)^{2}+\mu_{2}\left(\frac{\partial \bar{w}}{\partial \eta}\right)^{2}\right), \\
N_{\eta}=\frac{1}{1-\mu_{1} \mu_{2}} \cdot\left(\frac{\partial v}{\partial \eta}+\mu_{1} \frac{\partial u}{\partial \xi}\right)+\frac{1}{2\left(1-\mu_{1} \mu_{2}\right)} \cdot\left(\left(\frac{\partial w}{\partial \eta}\right)^{2}+\mu_{1}\left(\frac{\partial w}{\partial \xi}\right)^{2}\right), \\
T=\frac{G}{E_{2}} \cdot\left(\frac{\partial u}{\partial \eta}+\frac{\partial v}{\partial \xi}\right)+\frac{G}{E_{2}} \cdot\left(\frac{\partial w}{\partial \eta} \frac{\partial w}{\partial \xi}\right) .
\end{gathered}
$$


The motion equations are supplemented by boundary conditions, a type of which is defined by a way of plate-edge fixing.

\section{Research method for nonlinear forced vibration of orthotropic plate}

As shown in references $[7,13,14]$, determination of eigenfunctions appropriate to linear vibrations of an orthotropic plate can be fulfilled practically for arbitrary geometry and enough general boundary conditions by $R$-functions method (RFM). Due to application of RFM, the eigenfunctions are founded in an analytical form. This fact allows using obtained eigenfunctions as a basis for solving the nonlinear tasks, in particular, problems of large amplitude of vibration plates.

Let us suppose that for an orthotropic plate the eigenfunctions appropriate to the frequencies of free linear vibrations plate are known (see Section 4). Let eigenfunctions $W_{1}(\xi, \eta), W_{2}(\xi, \eta)$ be appropriated to the first two frequencies. It is supposed that lateral load may be presented as

$$
q(x, y, t)=P(t) \cdot W_{1}(x, y) .
$$

The deflection function is represented in the following form:

$$
W(\xi, \eta, t)=y_{1}(t) W_{1}(\xi, \eta)+y_{2}(t) W_{2}(\xi, \eta)
$$

After substitution of (3.2) into the first two equations (2.1), (2.2), one receives

$$
\begin{aligned}
& L_{11} u+L_{12} v=y_{1}^{2}(t) N l_{1}\left(W_{1}\right)+y_{2}^{2}(t) N l_{1}\left(W_{2}\right)+y_{1}(t) y_{2}(t) N l_{1} l_{1}\left(W_{1}, W_{2}\right), \\
& L_{21} u+L_{22} v=y_{1}^{2}(t) N l_{2}\left(W_{1}\right)+y_{2}^{2}(t) N l_{2}\left(W_{2}\right)+y_{1}(t) y_{2}(t) N l_{1} l_{2}\left(W_{1}, W_{2}\right),
\end{aligned}
$$

where the operators $N l_{1}\left(W_{i}\right), N l_{2}\left(W_{i}\right)(i=1,2)$ are defined by expressions (2.5), (2.6), and the operators $N l_{1} l_{1}\left(W_{1} W_{2}\right), N l_{2} l_{2}\left(W_{1} W_{2}\right)$ are represented below:

$$
\begin{aligned}
& N l_{1} l_{1}\left(W_{1} W_{2}\right) \\
& =-\left(\frac{\partial}{\partial \xi}\left(C_{1} \frac{\partial W_{1}}{\partial \xi} \frac{\partial W_{2}}{\partial \eta}+v_{1} \frac{\partial W_{1}}{\partial \eta} \frac{\partial W_{2}}{\partial \eta}\right)+C_{2} \frac{\partial}{\partial \eta}\left(\frac{\partial W_{1}}{\partial \xi} \frac{\partial W_{2}}{\partial \eta}+\frac{\partial W_{2}}{\partial \xi} \frac{\partial W_{1}}{\partial \eta}\right)\right), \\
& N l_{2} l_{2}\left(W_{1}, W_{2}\right) \\
& =-\left(\frac{\partial}{\partial \eta}\left(\frac{\partial W_{1}}{\partial \eta} \frac{\partial W_{2}}{\partial \eta}+v_{1} \frac{\partial W_{1}}{\partial \xi} \frac{\partial W_{2}}{\partial \xi}\right)+C_{2} \frac{\partial}{\partial \xi}\left(\frac{\partial W_{2}}{\partial \xi} \frac{\partial W_{1}}{\partial \eta}+\frac{\partial W_{1}}{\partial \xi} \frac{\partial W_{2}}{\partial \eta}\right)\right) .
\end{aligned}
$$


4 Research of nonlinear vibrations of orthotropic plates

Equations (3.3) will be satisfied identically, if the functions $u(\xi, \eta, t)$ and $v(\xi, \eta, t)$ are selected as

$$
\begin{aligned}
& u(\xi, \eta, t)=u_{1}(\xi, \eta) y_{1}^{2}(t)+u_{2}(\xi, \eta) y_{2}^{2}(t)+u_{3}(\xi, \eta) y_{1}(t) y_{2}(t), \\
& v(\xi, \eta, t)=v_{1}(\xi, \eta) y_{1}^{2}(t)+v_{2}(\xi, \eta) y_{2}^{2}(t)+v_{3}(\xi, \eta) y_{1}(t) y_{2}(t),
\end{aligned}
$$

where $\left(u_{1}, v_{1}\right),\left(u_{2}, v_{2}\right)$, and $\left(u_{3}, v_{3}\right)$ are solutions of the following set of equations:

$$
\begin{gathered}
L_{11} u_{1}+L_{12} v_{1}=N l_{1}\left(W_{1}\right), \\
L_{21} u_{1}+L_{22} v_{1}=N l_{2}\left(W_{1}\right), \\
L_{11} u_{2}+L_{12} v_{2}=N l_{1}\left(W_{2}\right), \\
L_{21} u_{2}+L_{22} v_{2}=N l_{2}\left(W_{2}\right), \\
L_{11} u_{3}+L_{12} v_{3}=N l_{1} l_{1}\left(W_{1}, W_{2}\right), \\
L_{21} u_{3}+L_{22} v_{3}=N l_{2} l_{2}\left(W_{1}, W_{2}\right) .
\end{gathered}
$$

Equations (3.6) are supplemented by appropriate boundary conditions. The obtained equations formally coincide with the equations of a plane problem in the theory of elasticity for an orthotropic body. Thus the expressions $N l_{1}\left(W_{1}\right), N l_{2}\left(W_{1}\right), N l_{1}\left(W_{2}\right), N l_{2}\left(W_{2}\right)$, $N l_{1} l_{1}\left(W_{1}, W_{2}\right)$, and $N l_{2} l_{2}\left(W_{1}, W_{2}\right)$ may be considered as mass forces. A solution of the plane problem in theory of elasticity for orthotropic plate is carried out by RFM as well (see Section 5). After finding solutions $\left(u_{1}, v_{1}\right),\left(u_{2}, v_{2}\right),\left(u_{3}, v_{3}\right)$, the functions (3.5) may be determined and substituted into (2.3) together with expressions for deflection (3.2) and load (3.1). As a result the ordinary differential equation is received. Let us apply method of Bubnov-Galerkin to the obtained equation. Projecting this equation on the eigenfunctions $W_{1}(\xi, \eta)$ and $W_{2}(\xi, \eta)$ and taking into account their orthogonality, one can receive the following system of the nonlinear differential equations:

$$
\begin{gathered}
y_{1}^{\prime \prime}+\alpha_{0} y_{1}+\alpha_{1} y_{1}^{3}+\alpha_{2} y_{1}^{2} y_{2}+\alpha_{3} y_{1} y_{2}^{2}+\alpha_{4} y_{2}^{3}=\alpha_{5} P(t), \\
y_{2}^{\prime \prime}+\beta_{0} y_{2}+\beta_{1} y_{1}^{3}+\beta_{2} y_{1}^{2} y_{2}+\beta_{3} y_{1} y_{2}^{2}+\beta_{4} y_{2}^{3}=0,
\end{gathered}
$$

where

$$
\begin{gathered}
\alpha_{0}=1, \quad \alpha_{1}=\frac{a_{13}}{\omega_{0}^{2}\left\|W_{1}\right\|^{2}}, \quad \alpha_{2}=\frac{a_{14}}{\omega_{0}^{2}\left\|W_{1}\right\|^{2}}, \\
\alpha_{3}=\frac{a_{15}}{\omega_{0}^{2}\left\|W_{1}\right\|^{2}}, \quad \alpha_{4}=\frac{a_{16}}{\omega_{0}^{2}\left\|W_{1}\right\|^{2}}, \quad \alpha_{5}=\frac{a_{17}}{\omega_{0}^{2}\left\|W_{1}\right\|^{2}}, \\
\beta_{0}=\frac{\omega_{2 L}^{2}}{\omega_{1 L}^{2}}, \quad \beta_{1}=\frac{a_{23}}{\omega_{0}^{2}\left\|W_{2}\right\|^{2}}, \quad \beta_{2}=\frac{a_{24}}{\omega_{0}^{2}\left\|W_{2}\right\|^{2}}, \\
\beta_{3}=\frac{a_{25}}{\omega_{0}^{2}\left\|W_{2}\right\|^{2}}, \quad \beta_{4}=\frac{a_{26}}{\omega_{0}^{2}\left\|W_{2}\right\|^{2}} .
\end{gathered}
$$


The expressions for coefficients $a_{i j}(i=1,2 ; j=0,1,2,3,4,5,6)$ in $(3.8)$ are

$$
\begin{aligned}
a_{i 1}= & \left\|w_{i}\right\|^{2}, \quad a_{i 2}=\lambda^{2} \omega_{i L}^{2}\left\|w_{i}\right\|^{2} \\
a_{i 3}= & -12\left(1-\mu_{1} \mu_{2}\right) \iint_{\Omega}\left(N_{x}\left(\vec{U}_{1}\right) \frac{\partial^{2} w_{1}}{\partial \xi^{2}}+2 T\left(\vec{U}_{1}\right) \frac{\partial^{2} w_{1}}{\partial \xi \partial \eta}+N_{y}\left(\vec{U}_{1}\right) \frac{\partial^{2} w_{1}}{\partial \eta}\right) w_{i} d \Omega, \\
a_{i 4}= & -12\left(1-\mu_{1} \mu_{2}\right) \iint_{\Omega}\left(N_{x}\left(\vec{U}_{1}\right) \frac{\partial^{2} w_{2}}{\partial \xi^{2}}+2 T\left(\vec{U}_{1}\right) \frac{\partial^{2} w_{2}}{\partial \xi \partial \eta}+N_{y}\left(\vec{U}_{1}\right) \frac{\partial^{2} w_{2}}{\partial \eta}\right) w_{i} d \Omega \\
& -12\left(1-\mu_{1} \mu_{2}\right) \iint_{\Omega}\left(N_{x}\left(\vec{U}_{3}\right) \frac{\partial^{2} w_{1}}{\partial \xi^{2}}+2 T\left(\vec{U}_{3}\right) \frac{\partial^{2} w_{1}}{\partial \xi \partial \eta}+N_{y}\left(\vec{U}_{3}\right) \frac{\partial^{2} w_{1}}{\partial \eta}\right) w_{i} d \Omega, \\
a_{i 5}= & -12\left(1-\mu_{1} \mu_{2}\right) \iint_{\Omega}\left(N_{x}\left(\vec{U}_{2}\right) \frac{\partial^{2} w_{1}}{\partial \xi^{2}}+2 T\left(\vec{U}_{2}\right) \frac{\partial^{2} w_{1}}{\partial \xi \partial \eta}+N_{y}\left(\vec{U}_{2}\right) \frac{\partial^{2} w_{1}}{\partial \eta}\right) w_{i} d \Omega \\
& -12\left(1-\mu_{1} \mu_{2}\right) \iint_{\Omega}\left(N_{x}\left(\vec{U}_{3}\right) \frac{\partial^{2} w_{2}}{\partial \xi^{2}}+2 T\left(\vec{U}_{3}\right) \frac{\partial^{2} w_{2}}{\partial \xi \partial \eta}+N_{y}\left(\vec{U}_{3}\right) \frac{\partial^{2} w_{2}}{\partial \eta}\right) w_{i} d \Omega, \\
a_{i 6}= & -12\left(1-\mu_{1} \mu_{2}\right) \iint_{\Omega}\left(N_{x}\left(\vec{U}_{2}\right) \frac{\partial^{2} w_{2}}{\partial \xi^{2}}+2 T\left(\vec{U}_{2}\right) \frac{\partial^{2} w_{2}}{\partial \xi \partial \eta}+N_{y}\left(\vec{U}_{2}\right) \frac{\partial^{2} w_{2}}{\partial \eta}\right) w_{i} d \Omega, \\
a_{17}=12\left(1-\mu_{1} \mu_{2}\right)\left\|w_{1}\right\| \|^{2} P(t), & a_{27}=0 .
\end{aligned}
$$

Solving the system (3.7), supplemented by the initial conditions

$$
y_{1}(0)=A_{\max }, \quad y_{1}^{\prime}(0)=0, \quad y_{2}(0)=0, \quad y_{2}^{\prime}(0)=0,
$$

can be carried out, for example, by Runge-Kutta method.

\section{Solving the linear vibrations problem of orthotropic plates}

Let us consider the problem of determination of the basis functions $W_{1}, W_{2}$ in more detail. For their finding, it is necessary to solve the problem of free linear vibrations of orthotropic plate; that is, it is necessary to solve the equation

$$
L_{31} W=\lambda^{2} \omega_{L}^{2} W
$$

The combination of variational method of Ritz and RFM $[12,13,15]$ is applied for solving the problem. According to RFM, it is necessary to make variational statement of the problem (4.1):

$$
\partial J=0,
$$


where

$$
\begin{aligned}
J(W)= & \iint_{\Omega}\left(C_{1}\left(\frac{\partial^{2} W}{\partial \xi^{2}}\right)^{2}+2 \mu_{1} \frac{\partial^{2} W}{\partial \xi^{2}} \frac{\partial^{2} W}{\partial \eta^{2}}+\left(\frac{\partial^{2} W}{\partial \eta^{2}}\right)^{2}+4 C_{2}\left(\frac{\partial^{2} W}{\partial \xi \partial \eta}\right)^{2}\right) \\
& \times \partial \Omega-\omega_{0}^{2} \iint_{\Omega} W^{2} \partial \Omega .
\end{aligned}
$$

Here $\omega_{0}^{2}=\lambda^{2} \omega_{L}^{2}$

A minimum of this functional is sought on a set of coordinate functions, which are constructed by the RFM. To form the sequences of the coordinate functions, it is necessary to construct the first structure of solution $[12,15]$ for boundary value problems (4.1). As shown in $[7,12,13,15]$, the solution structure depends on the type of boundary conditions. For example, the structure of solution, which satisfies to boundary conditions for clamped plate, is determined by the expression

$$
w=\omega^{2} P_{1}
$$

Here $P_{1}$ is an indefinite component of structure $[7,12,13,15]$. Note that all boundary conditions are satisfied independently of $P_{1}$ choice. Equation of domain boundary $\omega(x, y)=0$ is constructed by $R$-functions as a uniform analytical expression. It should be noted that the constructed equation $\omega(x, y)=0$ contains only elementary functions. The function $\omega(x, y)$ also satisfies the following conditions:

$$
\begin{array}{cl}
\omega(x, y)=0, & \forall(x, y) \in \partial \Omega, \\
\omega(x, y)>0, & \forall(x, y) \in \Omega, \\
\frac{\partial \omega(x, y)}{\partial n}=1, & \forall(x, y) \in \partial \Omega .
\end{array}
$$

In the case of simply supported edge of orthotropic plate, the structure of a solution is the following $[7,13]$ :

$$
w=\omega P_{1}-\frac{\omega^{2}}{2\left(A_{1}-\omega\right)}\left(A_{1}\left\{2 D_{1}^{(\omega)} P_{1}+P_{1} D_{2}^{(\omega)} \omega\right\}+2 A_{2} T_{1}^{(\omega)} P_{1}-\frac{1}{\rho} A_{3} P_{1}\right)+\omega^{3} P_{2},
$$

where $\rho$ is a continuation of a boundary curvature inside of the domain, and the operators $D_{m}^{(\omega)}, T_{m}^{(\omega)}(m=1,2)$ are defined as [12]

$$
\begin{gathered}
D_{m}^{(\omega)} f=\left(\frac{\partial \omega}{\partial x} \frac{\partial}{\partial x}+\frac{\partial \omega}{\partial y} \frac{\partial}{\partial y}\right)^{m} \cdot f \\
T_{m}^{(\omega)} f=\sum(-1)^{m-i} C_{m}^{i} \frac{\partial^{m} f}{\partial x^{m-i} \partial y^{i}}\left(\frac{\partial \omega}{\partial x}\right)^{i}\left(\frac{\partial \omega}{\partial y}\right)^{m-i}
\end{gathered}
$$


The coefficients $A_{1}, A_{2}, A_{3}$ are defined by the following expressions:

$$
\begin{aligned}
& A_{1}=-\left[D_{1}\left(\frac{\partial \omega}{\partial x}\right)^{4}+D_{2}\left(\frac{\partial \omega}{\partial y}\right)^{4}+2 D_{3}\left(\frac{\partial \omega}{\partial x}\right)^{2}\left(\frac{\partial \omega}{\partial y}\right)^{2}\right], \\
& A_{2}=\left[\left(D_{1}-D_{3}\right)\left(\frac{\partial \omega}{\partial x}\right)^{2}+\left(D_{3}-D_{2}\right)\left(\frac{\partial \omega}{\partial y}\right)^{2}\right]\left(\frac{\partial \omega}{\partial x}\right)\left(\frac{\partial \omega}{\partial y}\right), \\
& A_{3}=-\mu_{2} D_{1}+\left[2 D_{3}-\left(D_{1}+D_{2}\right)\right]\left(\frac{\partial \omega}{\partial x}\right)^{2}\left(\frac{\partial \omega}{\partial y}\right)^{2},
\end{aligned}
$$

where $D_{1}, D_{2}, D_{3}$ are rigidity coefficients, which are defined by the following formulas:

$$
D_{1}=\frac{E_{1} h^{3}}{12\left(1-\mu_{1} \mu_{2}\right)}, \quad D_{2}=\frac{E_{2} h^{3}}{12\left(1-\mu_{1} \mu_{2}\right)}, \quad D_{3}=D_{1} \mu_{2}+2 D_{k}, \quad D_{k}=\frac{G h^{3}}{12} \text {. }
$$

In the case when the plate is simply supported, it is possible to use the structure of solution which takes into account only principal (kinematic) conditions. For orthotropic and isotropic plates this solution structure can be represented in the following form:

$$
w=\omega P_{1}
$$

where the functions $P_{1}$ and $\omega(x, y)$ have the same sense, as earlier in the formulas $(4.4)$, (4.5).

If the plate is clamped on one part of boundary $\partial \Omega_{1}$ and is simply supported on the remaining part of the boundary $\partial \Omega_{2}=\partial \Omega \backslash \partial \Omega_{1}$, the solution structure satisfying only main conditions is [13] the following:

$$
w(x, y)=\omega \omega_{1} P_{1}
$$

where $\omega=0$ is an equation all over the boundary; $\omega_{1}=0$ is an equation of the clamped edge of the domain. Further components $P_{1}\left(P_{2}\right)$ in the structural formulas are expanded in a series of some complete set of functions $\left\{\psi_{k}\right\}$ : degree polynomials, trigonometric, splines, polynomials of Chebyshev, and so forth; that is,

$$
P_{i}=\sum_{k=1}^{n} a_{k}^{(i)} \psi_{k}
$$

After substitution of (4.12) into the structural formulas, the unknown function $W$ will be received in an analytical form. For example, for the clamped plate,

$$
W=\sum_{k=1}^{n} a_{k} \omega_{1}^{2} \psi_{k}=\sum_{k=1}^{n} a_{k} \varphi_{k}
$$

where $\varphi_{k}=\omega^{2} \psi_{k}$ is a complete set of coordinate functions satisfying the given boundary conditions, and $a_{k}, k=1,2, \ldots, n$, are unknown constant coefficients, which may be 
8 Research of nonlinear vibrations of orthotropic plates

founded from a condition of a functional minimum (4.2), that is, as a result of solving the linear algebraic equations system

$$
\frac{\partial J}{\partial a_{i}}=0, \quad i=1,2, \ldots, n
$$

\section{Solving the plane problem in the theory of elasticity by RFM}

Let us consider a method of solving a plane problem in the theory of elasticity for definition of functions $\left(u_{1}, v_{1}\right),\left(u_{2}, v_{2}\right),\left(u_{3}, v_{3}\right)$, that is, solving (3.6). The variational statement of the problem is fulfilled by Lagrange variational principle [10]. The functional by Lagrange reaches its minimum on the solution of the posed problem, that is,

$$
\partial I(\vec{U})=0 .
$$

In the extended form the functional $I(\vec{U})$ for displacements can be written as

$$
\begin{aligned}
I\left(\vec{U}_{i}\right)= & \iint_{\Omega}\left(C_{1}\left(\frac{\partial u_{i}}{\partial \xi}\right)^{2}+\left(\frac{\partial v_{i}}{\partial \eta}\right)^{2}+C_{2}\left(\frac{\partial v_{i}}{\partial \xi}+\frac{\partial u_{i}}{\partial \eta}\right)^{2}+2 \mu_{1} \frac{\partial u_{i}}{\partial \xi} \frac{\partial v_{i}}{\partial \eta}\right) d \Omega \\
& +I_{r}\left(\vec{U}_{i}\right)+I_{k}\left(\vec{U}_{i}\right)
\end{aligned}
$$

where $\vec{U}_{i}=\left(u_{i}, v_{i}\right)(i=1,2,3)$. Expressions for $I_{r}\left(\vec{U}_{i}\right), I_{k}\left(\vec{U}_{i}\right)$, in case $i=1,2$, are

$$
\begin{gathered}
I_{r}\left(\vec{U}_{i}\right)=2 \iint_{\Omega}\left(N l_{1}\left(W_{i}\right) u_{i}+N l_{2}\left(W_{i}\right) v_{i}\right) \partial \Omega, \\
I_{k}\left(\vec{U}_{i}\right)=\oint_{\partial \Omega}\left(C_{1}\left(l^{2}+\mu_{2} m^{2}\right)\left(\frac{\partial W_{i}}{\partial \xi}\right)^{2}+\left(m^{2}+\mu_{1} l^{2}\right)\left(\frac{\partial W_{i}}{\partial \eta}\right)^{2}+2 C_{2} \operatorname{lm} \frac{\partial W_{i}}{\partial \xi} \frac{\partial W_{i}}{\partial \eta}\right) u_{i n} \partial s \\
+\oint_{\partial \Omega}\left(C_{1}\left(\mu_{2}-1\right) \operatorname{lm}\left(\frac{\partial W_{i}}{\partial \xi}\right)^{2}+\left(1-\mu_{1}\right) \operatorname{lm}\left(\frac{\partial W_{i}}{\partial \eta}\right)^{2}\right. \\
\left.+2 C_{3}\left(l^{2}-m^{2}\right) \frac{\partial W_{i}}{\partial \xi} \frac{\partial W_{i}}{\partial \eta}\right) v_{i n} \partial s .
\end{gathered}
$$

For $i=3$,

$$
\begin{gathered}
I_{r}\left(\vec{U}_{3}\right)=2 \iint_{\Omega}\left(N l_{1} l_{1}\left(W_{1}, W_{2}\right) u_{3}+N l_{2} l_{2}\left(W_{1}, W_{2}\right) v_{3}\right) \partial \Omega \\
I_{k}\left(\vec{U}_{3}\right)=\oint_{\partial \Omega}\left(2 C_{1}\left(l^{2}+\mu_{2} m^{2}\right) \frac{\partial W_{1}}{\partial \xi} \frac{\partial W_{2}}{\partial \xi}+2\left(m^{2}+\mu_{1} l^{2}\right) \frac{\partial W_{1}}{\partial \eta} \frac{\partial W_{2}}{\partial \eta}\right) u_{i n} \partial s \\
+\oint_{\partial \Omega}\left(2 C_{1}\left(\mu_{2}-1\right) \operatorname{lm} \frac{\partial W_{1}}{\partial \xi} \frac{\partial W_{2}}{\partial \xi}+2\left(1-\mu_{1}\right) \operatorname{lm} \frac{\partial W_{1}}{\partial \eta} \frac{\partial W_{2}}{\partial \eta}\right. \\
\left.+2 C_{3}\left(l^{2}-m^{2}\right)\left(\frac{\partial W_{1}}{\partial \xi} \frac{\partial W_{2}}{\partial \eta}+\frac{\partial W_{2}}{\partial \xi} \frac{\partial W_{1}}{\partial \eta}\right)\right) v_{i n} \partial s
\end{gathered}
$$

where $u_{i n}=u_{i} l+v_{i} m, v_{i n}=-u_{i} m+v_{i} l$. 
Here $l$ and $m$ are directional cosines of a normal vector to the boundary $\partial \Omega$ of the domain $\Omega$. While calculating the directional cosines are substituted by the expressions

$$
l=-\omega^{\prime} 1 ; \quad m=-\omega^{\prime},
$$

where $\omega(x, y)=0$ is the normalized equation of the boundary, that is, the function $\omega(x, y)$ satisfies conditions (4.5).

Let us remark that in case of clamped immovable edge the tangent displacement on the boundary is equal to zero; therefore contour integral $I_{k}\left(\vec{U}_{i}\right)$ in a functional (5.2) will vanish.

The descretization of the functional is carried out on a set of functions satisfying, at least, kinematics boundary conditions. In case of a clamped edge the structural formulas are

$$
u_{i}=\omega P_{u}^{i}, \quad v_{i}=\omega P_{v}^{i}, \quad i=1,2,3 .
$$

The indefinite components $P_{u}^{i}, P_{v}^{i}$, as in solving the task on eigenvalues, are expanded in a series of some complete set of functions. The coefficients of these expansions are founded from condition of a functional minimum (5.2), that is, as a result of solving the linear algebraic equations system.

The proposed investigation method of nonlinear free vibrations of orthotropic plates with an arbitrary shape was realized with "POLE-RL" program system $[15,16]$, designed at AN Podgorny Institute for Mechanical Engineering Problems of NAS of Ukraine under guidance of the academician Rvachev.

\section{Numerical results}

Example 6.1. The proposed algorithm was tested for simply supported and clamped square plate with immovable edge. The boundary conditions for functions $u, v$ over the boundary were accepted as follows:

$$
u=0, \quad v=0, \quad \forall(x, y) \in \partial \Omega .
$$

In case of a clamped edge, the boundary conditions for deflection functions are

$$
w=0, \quad \frac{\partial w}{\partial n}=0
$$

in case of a simply supported edge,

$$
w=0, \quad M_{n}=0 .
$$

Let the plates be manufactured of glass-epoxy and aragonite crystal materials (Table $6.1)$.

The obtained results for different materials and for isotropic plates are compared with similar results that were obtained earlier and presented in $[3,6,9,10,17]$. This comparison is presented in Tables 6.2 and 6.3. 
Table 6.1

\begin{tabular}{lllc}
\hline Material & $E_{2} / E_{1}$ & $G_{12} / E_{2}$ & $\nu_{12}=\nu_{21} E_{2} / E_{1}$ \\
\hline Glass epoxy (A) & 3 & 0.5 & 0.25 \\
Aragonite crystal (B) & 0.543103 & 0.262931 & 0.23319 \\
\hline
\end{tabular}

Table 6.2. Nonlinear free-vibration frequency ratio $\omega_{N} / \omega_{L}$ for a simply supported isotropic square plate.

\begin{tabular}{ccccccc}
\hline$w / h$ & {$[3]$} & {$[6]$} & {$[10]$} & {$[17]$} & {$[9]$} & RFM \\
\hline 0.2 & 1.03 & 1.02 & 1.02 & 1.02 & 1.01 & 1.03 \\
0.4 & 1.10 & 1.10 & 1.09 & 1.07 & 1.05 & 1.08 \\
0.6 & 1.21 & 1.21 & 1.20 & 1.15 & 1.12 & 1.19 \\
0.8 & 1.35 & 1.35 & 1.34 & 1.27 & 1.20 & 1.32 \\
1.0 & 1.52 & 1.51 & 1.51 & 1.38 & 1.30 & 1.52 \\
\hline
\end{tabular}

Table 6.3. Nonlinear free-vibration frequency ratio $\omega_{N} / \omega_{L}$ for a simply supported orthotropic square plate.

\begin{tabular}{c|cc|cc}
\hline$w / h$ & \multicolumn{2}{|c|}{ Material A } & \multicolumn{2}{c}{ Material B } \\
& {$[6]$} & RFM & {$[6]$} & RFM \\
\hline 0.2 & 1.03 & 1.03 & 1.03 & 1.03 \\
0.4 & 1.11 & 1.10 & 1.10 & 1.09 \\
0.6 & 1.23 & 1.23 & 1.21 & 1.20 \\
0.8 & 1.39 & 1.41 & 1.36 & 1.35 \\
1.0 & 1.57 & 1.65 & 1.52 & 1.55 \\
\hline
\end{tabular}

Example 6.2. To illustrate the resources of the proposed method, the task of nonlinear vibrations of a complex planform plate (Figure 6.1) is considered.

Let us consider two cases (clamped and simply supported) of boundary conditions and also two types of materials. The elastic constants of these materials are presented in Table 6.1. To concretize the structural formulas (4.4), (4.10), and (5.6), the equation of domain boundary is constructed by $R$-functions. It is easy to check that the function $\omega(x, y)$ can be represented as

$$
\omega(x, y)=\left(F_{1} \wedge_{0} F_{2}\right) \wedge_{0}\left(F_{3} \vee_{0} F_{4}\right) \wedge_{0}\left(F_{5} \vee_{0} F_{6}\right)
$$

where the functions $F_{i}(i=1,2,3,4,5,6)$ are defined by the following analytical expressions:

$$
\begin{array}{cl}
F_{1}=\frac{a^{2}-x^{2}}{2 a} \geq 0, & F_{2}=\frac{b^{2}-y^{2}}{2 b} \geq 0, \\
F_{3}=d_{2}+y \geq 0, \quad F_{4}=c_{2}+x \geq 0, & F_{5}=d_{1}-y \geq 0, \quad F_{6}=c_{1}-x \geq 0 .
\end{array}
$$




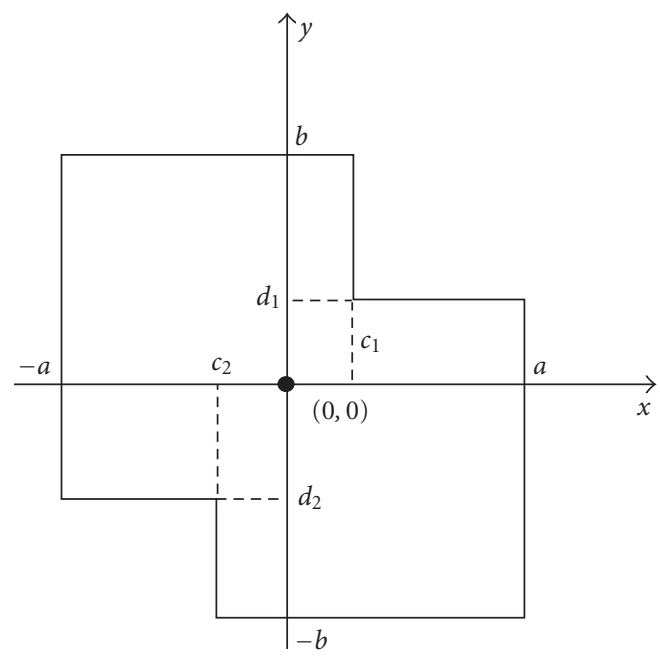

Figure 6.1

In the formula (6.4) the symbols $\wedge_{0}, \vee_{0}$ are signs of $R$-operations [12], defined according to the formulas

$$
X \wedge_{0} Y=X+Y-\sqrt{X^{2}+Y^{2}}, \quad X \vee_{0} Y=X+Y+\sqrt{X^{2}+Y^{2}} .
$$

It is easy to check that the constructed function $\omega(x, y)$ satisfies conditions (4.5). For approximation of the indefinite components in the constructed structural formulas, it is possible to use a system of degree polynomials

$$
1, x, y, x^{2}, x y, y^{2}, x^{3}, x^{2} y, x y^{2}, y^{3}, x^{4}, x^{3} y, \ldots
$$

In Table 6.4 the obtained results for linear fundamental frequencies are presented for the given plate at variation of the ratio $\alpha=c_{1} / 2 a=c_{2} / 2 a=d_{1} / 2 a=d_{2} / 2 a$ and a number of coordinate functions $(n=\mathrm{NCF})$ approximating the deflection $W$ in expression (4.13). If parameter $\alpha \rightarrow 1$, then the form of the plate shown in Figure 6.1 tends to square form. To research practical convergence of the obtained results, the different degree of approximating polynomials was chosen. It was established that for solving linear vibrations problem, the tenth degree of polynomials that corresponds to 66 of the coordinate functions for $W$, may be used. A further increase of coordinate functions number does not change the obtained results in the third sign after comma.

The amplitude-frequency dependence for the clamped orthotropic plates, which have the following relations of geometrical parameters: $b / a=1 ; c_{1} / a=c_{2} / a=1 / 2 ; d_{1} / a=$ $d_{2} / a=1 / 2$, is represented in Table 6.5.

The amplitude-frequency dependence of a simply supported plate with immovable plane edge for the same geometrical sizes of plate (Figure 6.1) is represented in Table 6.6.

To check the reliability of the result obtained for amplitude-frequency dependence of the plate with complex form, let us carry out series of calculations for this plate (Figure 6.1), for instance, manufactured from material $B$ with clamped boundary condition, 
12 Research of nonlinear vibrations of orthotropic plates

Table 6.4. Convergence of a nondimensional linear frequency parameter $\Lambda=\omega_{11}^{0} \cdot a^{2}\left(\rho / E_{2} h^{2}\right)^{1 / 2}$ of fundamental mode for orthotropic plate with complex form (Figure 6.1).

\begin{tabular}{|c|c|c|c|c|c|}
\hline \multirow{2}{*}{ Material } & \multirow{2}{*}{$\mathrm{NCF}$} & \multicolumn{4}{|c|}{$\alpha$} \\
\hline & & 0.25 & 0.4 & 0.45 & 1 (square) \\
\hline \multicolumn{6}{|c|}{ Clamped } \\
\hline \multirow{3}{*}{ A } & 55 & 11.294 & 9.212 & 9.174 & 9.171 \\
\hline & 66 & 11.291 & 9.204 & 9.173 & 9.171 \\
\hline & 91 & 11.291 & 9.204 & 9.173 & 9.171 \\
\hline \multirow{3}{*}{ B } & 55 & 15.437 & 12.370 & 12.322 & 12.320 \\
\hline & 66 & 15.423 & 12.367 & 12.321 & 12.320 \\
\hline & 91 & 15.423 & 12.367 & 12.321 & 12.319 \\
\hline \multirow{3}{*}{ Isotropic } & 55 & 13.697 & 10.935 & 10.891 & 10.889 \\
\hline & 66 & 13.691 & 10.930 & 10.890 & 10.847 \\
\hline & 91 & 13.691 & 10.930 & 10.890 & 10.847 \\
\hline \multicolumn{6}{|c|}{ Simply supported } \\
\hline \multirow{3}{*}{ A } & 55 & 7.560 & 5.166 & 5.004 & 4.871 \\
\hline & 66 & 7.488 & 5.158 & 4.997 & 4.871 \\
\hline & 91 & 7.488 & 5.158 & 4.997 & 4.871 \\
\hline \multirow{3}{*}{ B } & 55 & 10.870 & 7.112 & 6.748 & 6.587 \\
\hline & 66 & 10.792 & 7.101 & 6.740 & 6.587 \\
\hline & 91 & 10.792 & 7.101 & 6.740 & 6.587 \\
\hline \multirow{3}{*}{ Isotropic } & 55 & 9.663 & 6.415 & 6.122 & 5.973 \\
\hline & 66 & 9.594 & 6.405 & 6.116 & 5.973 \\
\hline & 91 & 9.594 & 6.405 & 6.116 & 5.973 \\
\hline
\end{tabular}

Table 6.5. Free-vibration frequency ratio $\omega_{N} / \omega_{L}$ for a clamped orthotropic plate with a complex form (Figure 6.1).

\begin{tabular}{lccc}
\hline$w / h$ & Material A & Material B & Isotropic \\
\hline 0.2 & 1.004 & 1.003 & 1.003 \\
0.4 & 1.018 & 1.011 & 1.011 \\
0.6 & 1.037 & 1.024 & 1.026 \\
0.8 & 1.065 & 1.043 & 1.045 \\
1.0 & 1.100 & 1.066 & 1.070 \\
1.2 & 1.142 & 1.094 & 1.099 \\
1.4 & 1.189 & 1.126 & 1.133 \\
1.6 & 1.241 & 1.161 & 1.171 \\
1.8 & 1.297 & 1.201 & 1.212 \\
2.0 & 1.357 & 1.243 & 1.257 \\
\hline
\end{tabular}

when parameter $\alpha=c_{1} / 2 a=c_{2} / 2 a=d_{1} / 2 a=d_{2} / 2 a$ approaches to 1 and the given plate takes planform of square plate. Results of these investigations are presented in Table 6.7. Example 6.3. The plate with the plan represented in Figure 6.2 is considered. The plate is under uniform load, which is changed in time by harmonic law. 
Table 6.6. Nonlinear free-vibration frequency ratio $\omega_{N} / \omega_{L}$ for a simply supported orthotropic plate (Figure 6.1).

\begin{tabular}{cccc}
\hline$w / h$ & Material A & Material B & Isotropic \\
\hline 0.2 & 1.011 & 1.008 & 1.008 \\
0.4 & 1.043 & 1.031 & 1.032 \\
0.6 & 1.095 & 1.069 & 1.071 \\
0.8 & 1.164 & 1.121 & 1.123 \\
1.0 & 1.246 & 1.184 & 1.187 \\
1.2 & 1.340 & 1.256 & 1.261 \\
1.4 & 1.444 & 1.336 & 1.343 \\
1.6 & 1.554 & 1.424 & 1.432 \\
1.8 & 1.671 & 1.516 & 1.526 \\
2.0 & 1.793 & 1.614 & 1.625 \\
\hline
\end{tabular}

Table 6.7. Nonlinear free-vibration frequency ratio $\omega_{N} / \omega_{L}$ for a clamped orthotropic plates (Figure 6.1, Material B).

\begin{tabular}{c|ccccc}
\hline \multirow{2}{*}{ w/h } & \multicolumn{5}{c}{$\alpha$} \\
\cline { 2 - 6 } & 0.25 & 0.4 & 0.45 & 0.49 & 1 \\
\hline 0.2 & 1.003 & 1.003 & 1.003 & 1.003 & 1.003 \\
0.4 & 1.011 & 1.014 & 1.014 & 1.014 & 1.016 \\
0.6 & 1.024 & 1.031 & 1.031 & 1.031 & 1.032 \\
0.8 & 1.043 & 1.054 & 1.054 & 1.054 & 1.057 \\
1.0 & 1.066 & 1.083 & 1.084 & 1.083 & 1.088 \\
1.2 & 1.094 & 1.118 & 1.118 & 1.118 & 1.124 \\
1.4 & 1.126 & 1.157 & 1.158 & 1.158 & 1.166 \\
1.6 & 1.162 & 1.201 & 1.202 & 1.202 & 1.212 \\
1.8 & 1.201 & 1.249 & 1.251 & 1.250 & 1.263 \\
2.0 & 1.243 & 1.301 & 1.303 & 1.302 & 1.317 \\
\hline
\end{tabular}

Let us consider three kinds of materials: glass epoxy, boron epoxy, and graphite epoxy. Physical elastic constants for these materials are presented in Table 6.8.

Calculation was carried out for two types of boundary conditions:

(a) clamped plate with movable edge:

$$
w=0, \quad \frac{\partial w}{\partial n}=0, \quad \frac{\partial^{2} \Phi}{\partial \tau^{2}}=0, \quad \frac{\partial^{2} \Phi}{\partial n \partial \tau}=0,
$$

where $\Phi$ is force function [19].

(b) simply supported plate with movable edge:

$$
W=0, \quad M_{n}=0, \quad \frac{\partial^{2} \Phi}{\partial \tau^{2}}=0, \quad \frac{\partial^{2} \Phi}{\partial n \partial \tau}=0 .
$$

The function $\omega(x, y)$ is constructed by $R$-functions [12]:

$$
\omega(x, y)=\left(f_{3} \vee_{0} f_{4}\right) \wedge_{0}\left(f_{1} \wedge_{0} f_{2}\right) \wedge_{0}\left(f_{5} \wedge_{0} f_{6}\right) .
$$


14 Research of nonlinear vibrations of orthotropic plates

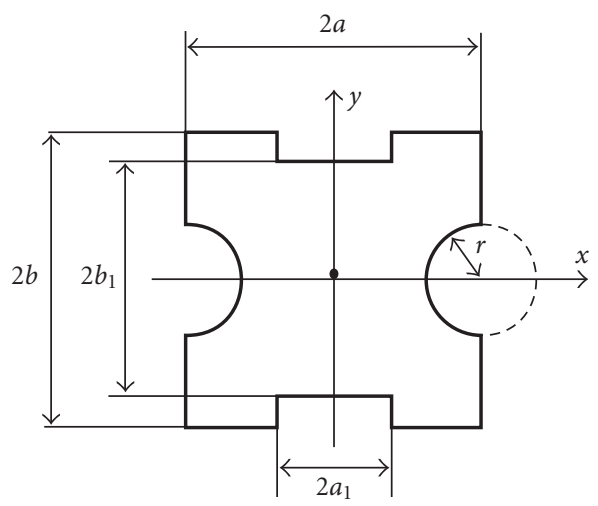

Figure 6.2. Planform of the plate.

Table 6.8

\begin{tabular}{lccc}
\hline Material & $E_{1} / E_{2}$ & $G_{12} / E_{2}$ & $\nu_{12}=\nu_{21} E_{2} / E_{1}$ \\
\hline Glass epoxy & 3 & 0.6 & 0.25 \\
Boron epoxy & 10 & $1 / 3$ & 0.22 \\
Graphite epoxy & 40 & 0.5 & 0.25 \\
\hline
\end{tabular}

Table 6.9. Linear frequency parameter, $\omega_{11}^{0} \cdot a^{2}\left(\rho / E_{2} h^{2}\right)^{1 / 2}$, of fundamental mode.

\begin{tabular}{lcc}
\hline Material & Clamped plate & Simply supported plate \\
\hline Glass epoxy & 21.2 & 14.6 \\
Boron epoxy & 32.3 & 18.7 \\
Graphite epoxy & 58.6 & 31.8 \\
Isotropic & 15.2 & 11.6 \\
\hline
\end{tabular}

Functions $f_{i}(i=1,2, \ldots, 6)$ are determined by the following expressions:

$$
\begin{gathered}
f_{1}=\frac{1}{2 a}\left(a^{2}-x^{2}\right), \quad f_{2}=\frac{1}{2 b}\left(b^{2}-y^{2}\right), \quad f_{3}=-\frac{1}{2 a_{1}}\left(a_{1}^{2}-x^{2}\right), \\
f_{4}=\frac{1}{2 b_{1}}\left(b_{1}^{2}-y^{2}\right), \quad f_{5}=\frac{1}{2 r}\left((x-a)^{2}+y^{2}-r^{2}\right), \\
f_{6}=\frac{1}{2 r}\left((x+a)^{2}+y^{2}-r^{2}\right) .
\end{gathered}
$$

There were chosen the following values of geometrical parameters of a plate: $b / a=1$; $b_{1} / a=0.375 ; a_{1} / a=0.125 ; r / a=0.125$. Values of the basic linear frequency for all kinds of material are presented in Table 6.9. The amplitude-frequency dependences for nonlinear free vibrations are presented in Figures 6.3 and 6.4. Numerical results were obtained using power polynomial approximation of the indefinite components. The approximate 


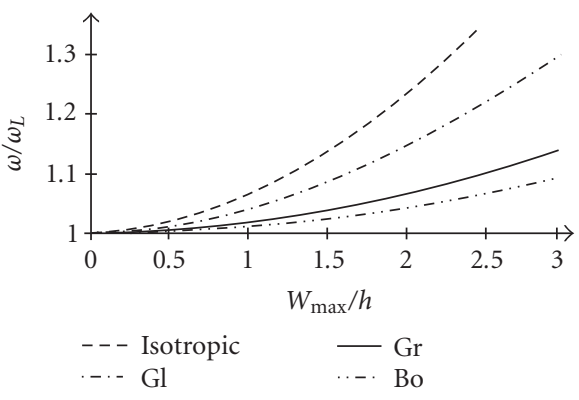

(a)

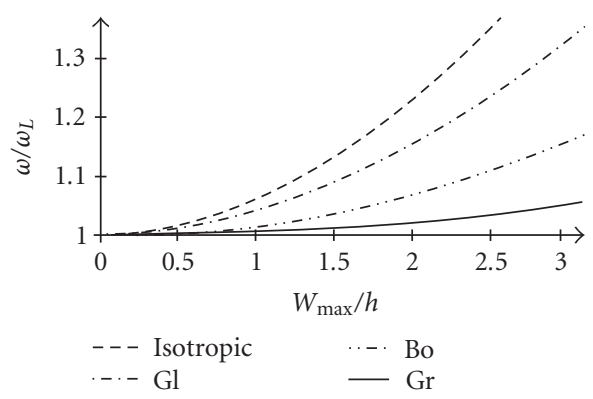

(b)

Figure 6.3. (a) Effect of elastic properties on nonlinear frequency of a clamped orthotropic plate. (b) Effect of elastic properties on nonlinear frequency of a simply supported orthotropic plate.

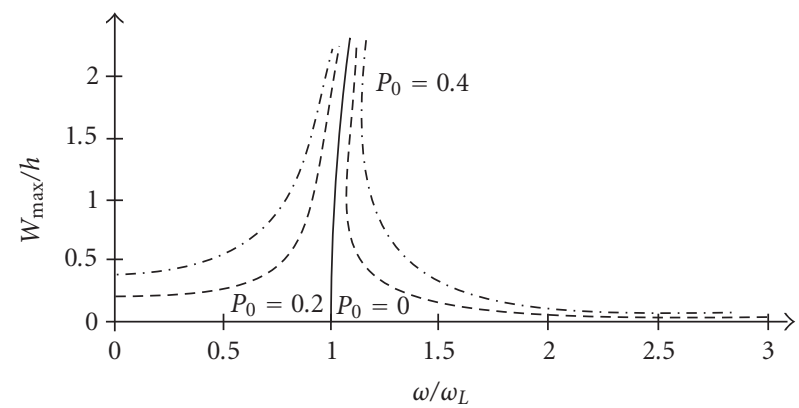

(a)

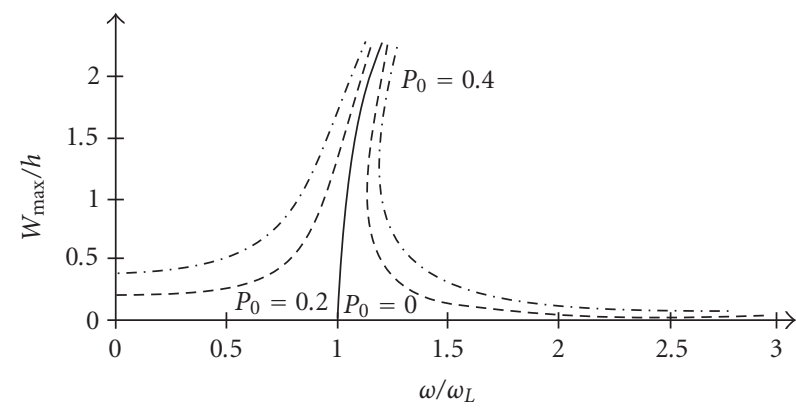

(b)

Figure 6.4. (a) Frequency response function for a clamped plate (graphite epoxy). (b)Frequency response function for a simply supported plate (glass epoxy).

polynomials were chosen up to 14 degrees which correspond to 45 terms of series. The symmetry of a problem was taken into account. Calculation of Ritz matrix elements was carried out by 10-dot Gauss's formulas. 


\section{Conclusion}

In this work, the research method for forced nonlinear vibrations of an arbitrary form orthotropic plates was proposed. This approach is based on $R$-functions theory, variational methods, and Runge-Kutt method. The software "POLE-RL" is applied to obtain the numerical results. The investigations are carried out for plates of different shapes, boundary conditions, and materials. The amplitude-frequency dependences are obtained and presented by graphics. The obtained results for a square plate are compared with known results. This comparison confirms effectiveness and reliability of the proposed method and created a software.

\section{References}

[1] S. A. Ambartsumyan, The General Theory of Anisotropic Shells, Izdat. "Nauka”, Moscow, 1974.

[2] C. Y. Chia, Nonlinear Analysis of Plates, McGraw-Hill, New York, 1980.

[3] H.-N. Chu and G. Herrmann, Influence of large amplitudes on free flexural vibrations of rectangular elastic plates, Journal of Applied Mechanics and Technical Physics 23 (1956), 532-540.

[4] P. C. Dumir and A. Bhaskar, Nonlinear forced vibration of orthotropic thin rectangular plates, International Journal of Mechanical Science 30 (1988), no. 5, 371-380.

[5] M. Ganapathi, T. K. Varadan, and B. S. Sarma, Nonlinear flexural vibrations of laminated orthotropic plates, Computers \& Structures 39 (1991), no. 6, 685-688.

[6] K. Kanaka Raju and E. Hinton, Nonlinear vibrations of thick plates using Mindlin plate elements, International Journal for Numerical Methods in Engineering. 15 (1980), no. 2, 249-257.

[7] L. V. Kurpa, V. L. Rvachev, and E. Ventsel, The R-function method for the free vibration analysis of thin orthotropic plates of arbitrary shape, Journal of Sound and Vibration 261 (2003), no. 1, 109-122.

[8] T. Manoj, M. Ayyappan, K. S. Krishnan, and B. Nageswara Rao, Nonlinear vibration analysis of thin laminated rectangular plates on elastic foundations, ZAMM. Zeitschrift für Angewandte Mathematik und Mechanik. Journal of Applied Mathematics and Mechanics 80 (2000), no. 3, 183-192.

[9] C. Mei and K. Decha-Umphai, A finite element method for nonlinear forced vibrations of rectangular plates, AIAA Journal 23 (1985), no. 7, 1104-1110.

[10] S. G. Mikhlin, Variational Methods in Mathematical Physics, 2nd ed., Izdat. "Nauka", Moscow, 1970.

[11] M. K. Prabhakara and C. Y. Chia, Non-linear flexural vibrations of orthotropic rectangular plates, Journal of Sound and Vibration 52 (1977), no. 4, 511-518.

[12] V. L. Rvachev, The Theory of R-Functions and Some of Its Applications, Naukova Dumka, Kiev, 1982.

[13] V. L. Rvachev and L. V. Kurpa, R-Functions in Problems of the Theory of Plates, Naukova Dumka, Kiev, 1987.

[14] Application of R-functions theory to plates and shells of a complex form, Journal of Mechanical Engineering (1998), no. 1, 33-53 (Russian).

[15] V. L. Rvachev and T. I. Sheiko, R-functions in boundary value problems in mechanics, Applied Mechanical Review 48 (1995), no. 4, 151-188.

[16] V. L. Rvachev and A. N. Shevchenko, The Problem-Orientation Languages and Systems for Engineering Research, Technica, Kiev, 1988.

[17] B. S. Sarma, Nonlinear free vibrations of beams, plates and nonlinear panel flutter, Ph.D. thesis, Department of Aerospace Engineering, I. I. T. Madras, Chennai, 1987.

[18] Y. Shi and C. Mei, A finite element time domain modal formulation for large amplitude free vibrations of beams and plates, Journal of Sound and Vibration 193 (1996), no. 2, 453-464. 
[19] A. S. Vol'mir, Flexible Plates and Shells, Gosudarstv. Izdat. Tehn.-Teor. Lit., Moscow, 1956. [20] , Nonlinear Dynamics of Plates and Shells, Izdat. "Nauka", Moscow, 1972.

L. V. Kurpa: Department of Applied Mathematics, National Polytechnic University KhPI, 21 Frunze Street, 61002 Kharkov, Ukraine E-mail address: kurpa@kpi.kharkov.ua

T. V. Shmatko: Department of Higher Mathematics, National Polytechnic University KhPI, 21 Frunze Street, 61002 Kharkov, Ukraine

E-mail address: ktv_ua@yahoo.com

O. G. Onufrienko: Department of Applied Mathematics, National Polytechnic University KhPI, 21 Frunze Street, 61002 Kharkov, Ukraine

E-mail address: onufrienko@mail.ru 


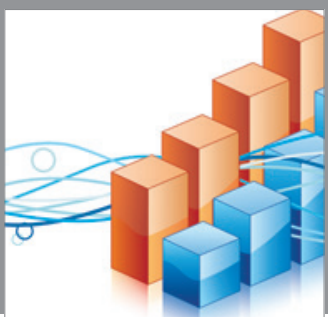

Advances in

Operations Research

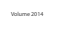

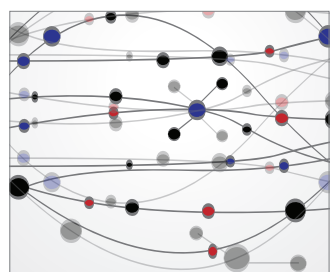

\section{The Scientific} World Journal
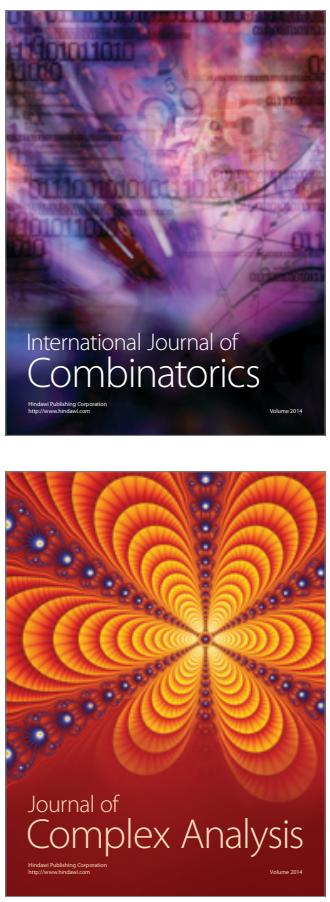

International Journal of

Mathematics and

Mathematical

Sciences
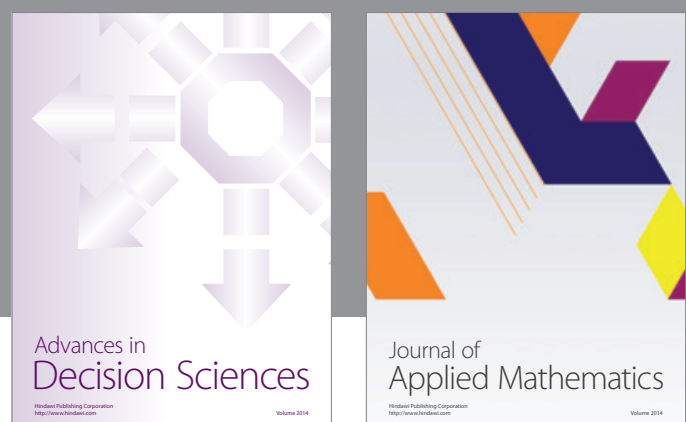

Journal of

Applied Mathematics
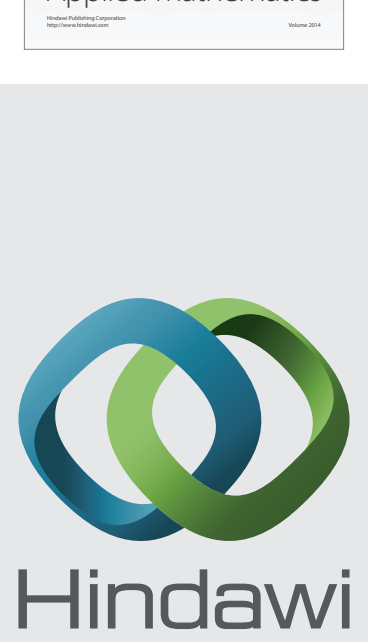

Submit your manuscripts at http://www.hindawi.com
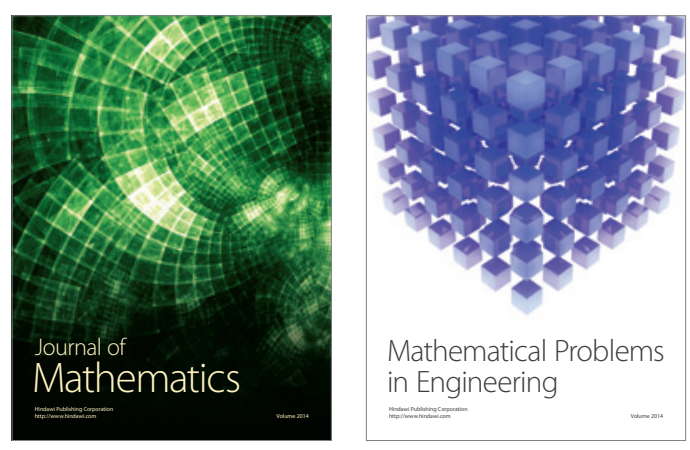

Mathematical Problems in Engineering
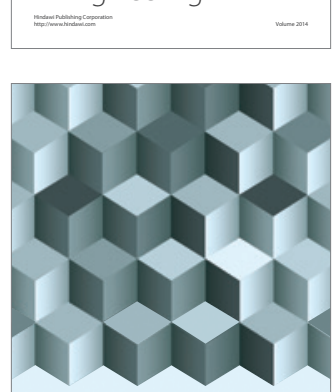

Journal of

Function Spaces
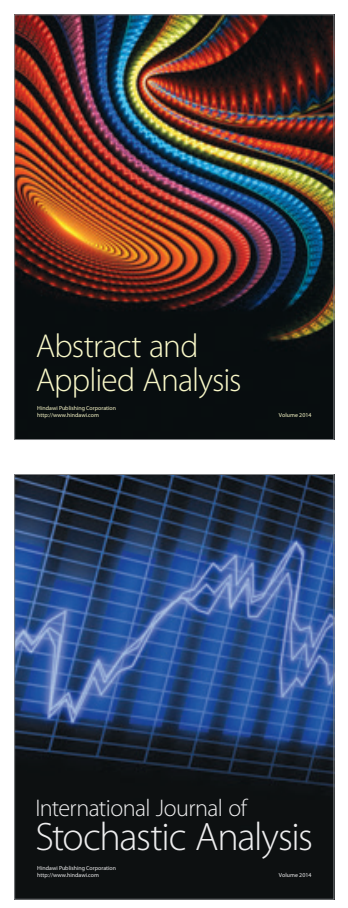

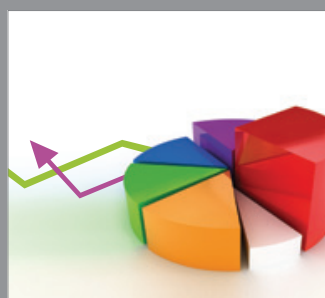

ournal of

Probability and Statistics

Promensencen
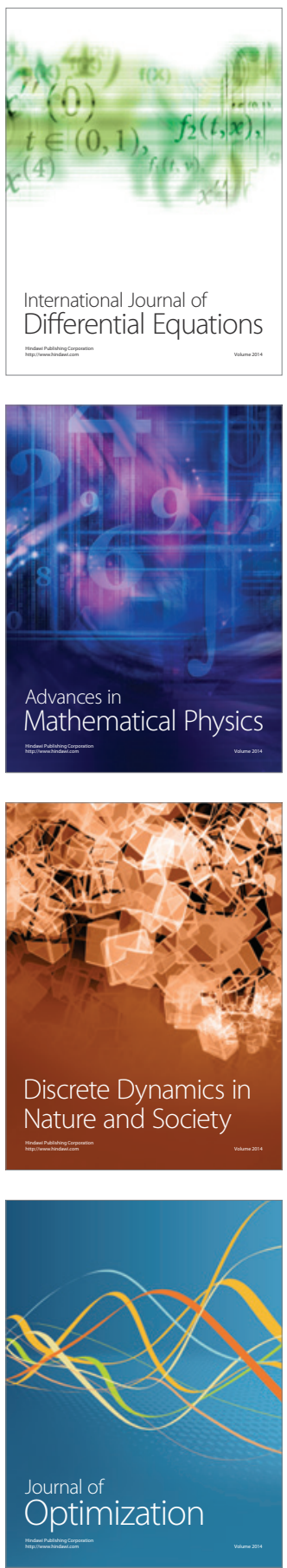